\title{
The Nanoscale Basis of Atrial Fibrillation: Functional Impact of Disrupting Nav1.5- rich Intercalated Disk Nanodomains.
}

Heather Struckman ${ }^{1}$, Amara Greer-Short ${ }^{2}$, Stephen Baine ${ }^{2}$, Louisa Mezache ${ }^{2}$, Anna Phillips ${ }^{2}$, Sandor Gyorke $^{2}$, Thomas Hund ${ }^{2}$ and Rengasayee Veeraraghavan ${ }^{2}$

${ }^{1}$ The Ohio State University, Hilliard, Ohio, United States, ${ }^{2}$ The Ohio State University, Columbus, Ohio, United States

Background: Atrial fibrillation (AF), which is characterized by chaotic patterns of electrical activation of the atria, affects over 4 million people in the US alone. We previously identified nanoscale structural abnormalities in the hearts of AF patients. Specifically, they displayed swelling of gap junction (GJ) adjacent perinexi, specialized nanodomains rich in cardiac sodium channels (Nav1.5) and located within intercalated disks (IDs; sites of electromechanical contact between adjacent cells). However, the functional consequences of these nanoscale structural changes remain unclear.

Objective: We assessed the structural and functional impacts of selectively disrupting different Nav1.5rich ID nanodomains.

Methods and Results: We utilized peptide mimetics of adhesion domains to selectively inhibit adhesion within different ID nanodomains: 1) Nadp1 (target: N-cadherin), 2) dadp1 (target: Desmoglein-2), and 3) $\beta$ adp1 (target: sodium channel $\beta 1$ subunit [SCN1b]). Each active peptide was compared against a corresponding inactive control peptide (Nadp1-c, dadp1-c, $\beta$ adp1-scr). Sub-diffraction confocal imaging revealed ID enrichment of active peptides, but not inactive controls. Furthermore, each active peptide was preferentially localized in ID regions rich in its corresponding protein target. Peptide treatment $(100 \mu \mathrm{M}$; 60 minutes) of ex vivo mouse hearts revealed profound widening of perinexi by $\beta$ adp 1 and of mechanical junctions by Nadp1. Dadp1 also induced widening of mechanical junctions albeit to a lower degree. STORM single molecule localization microscopy identified about $50 \%$ of ID-localized Nav1.5 within GJadjacent perinexi, while an additional $\sim 35 \%$ was located within $\mathrm{N}$-cad-rich ID sites. Nadp1 and $\beta$ adp1 induced redistribution of ID localized Nav1.5 away from perinexi and mechanical junctions respectively. Dadp1, again, had similar but milder effects compared to Nadp1. Western blot revealed the expression levels of Nav1.5, connexin 43 (Cx43), connexin $40(\mathrm{Cx} 40), \beta 1$ in peptide treated hearts to be within 10\% of levels in untreated controls. Optical mapping revealed atrial conduction slowing in hearts treated with Nadp1 $(17 \mathrm{~cm} / \mathrm{s}, 70.83 \%$ of control) and $\beta \operatorname{adp} 1(13 \mathrm{~cm} / \mathrm{s}, 54.17 \%$ of control), but not inactive control peptides $(24 \mathrm{~cm} / \mathrm{s})$. Volume-conducted electrocardiograms $(\mathrm{ECG})$ revealed $\mathrm{P}$ wave prolongation in active peptide treated hearts (Nadp1: 26.5ms, $\beta \mathrm{adp} 1: 31 \mathrm{~ms}$ ), consistent with conduction slowing compared to the inactive control peptides (16ms). Importantly, burst pacing elicited atrial arrhythmias in all hearts treated with Nadp1 and ßadp1. Arrhythmia burden (duration, number of arrhythmias) was highest with ßadp1.

Conclusions: These results suggest that disruption of Nav1.5-rich ID nanodomains impairs electrical impulse propagation and promotes arrhythmias in the atria. Furthermore, the magnitude of functional impacts are likely determined by the amount of sodium channels contained within the nanodomains disrupted. 